\section{Testverfahren in der neurologischen Physio- und Ergotherapie}

\author{
Assessments und einfache objektive Tests sind bei der Behandlung von neurologisch \\ erkrankten Patienten nicht mehr wegzudenken. Zum einen bieten sie Aufschluss über \\ den Effekt einer Behandlung, zum anderen sind sie ein wichtiger Motivationsfaktor für \\ den Patienten und wegweisend für die weiterführende Behandlung und Argumentation \\ des Therapeuten. Doch welche Testverfahren erweisen sich im klinischen Alltag als \\ praktikabel? \\ Simone Thomas, Bettina Scheffler, Bernhard Elsner, Jan Mehrholz
}

Testverfahren sind hilfreich und wichtig. Jedoch ist es schwer, aus der Vielzahl von Skalen, Fragebögen und Scores den idealen Test für den Patienten und für die eigene Arbeitssituation herauszufiltern. Vor allem für Patienten nach Schlaganfall existiert eine Vielzahl an Testverfahren.

Die American Heart Association hat dazu einen interessanten Artikel zu den wichtigsten Assessments bei Schlaganfallpatienten verfasst [45]. Einige dieser dort genannten Assessments benötigen allerdings sehr viel Zeit, Materialien und Aufwand, wie z.B. der Action-Research-Arm-Test oder der FuglMeyer-Test. Daher werden diese Verfahren häufig in Studien verwendet. Für den klinischen Alltag bleibt beim Testen meist wenig Zeit, weshalb hier gern schnelle und einfache Assessments verwendet werden sollten (hohe Praktikabilität). Diese Tests können nach der Einteilung der International Classification of Functioning, Disability and Health (ICF) Folgendes messen:

- Körperfunktionen

- Aktivitäten

- Teilhabe am alltäglichen Leben

In diesem Artikel werden für jede Ebene mögliche Assessments ausführlich vorgestellt.

\section{Mögliche Assessments in der Neurorehabilitation}

Im klinischen Alltag der Neurorehabilitation ist eine ganze Reihe von Assessments bekannt. Aber welche sind zuverlässig und für welche klinischen Situationen geeignet?

Um das richtige Assessment zu finden, bietet es sich an, dieses nach der individuellen Ziel- bzw. Problemstellung zu wählen:

- Ist es das Ziel, sicher und ohne Störung des Gleichgewichts gehen zu können, eignet sich z. B. der Dynamic Gait Index [53].

- Treten Probleme mit der Balance bereits im Stand auf, können die Berg Balance
Scale oder der Functional-Reach-Test den Verlauf objektiv darstellen [6, 16].

- Um das feinmotorische Greifen zu beurteilen, kann der Nine-Hole-Peg-Test und für grobmotorische Aktivitäten der Box-and-Block-Test verwendet werden [36, 37].

Neben diesen spezifischen Tests existieren weiterhin komplette Mobilitätsindexe, wie z. B. die Motor Assessment Scale [9]. Damit können spezifische Defizite und Ressourcen des Patienten ermittelt und Verbesserungen bei globaler Betrachtung des Patienten festgehalten werden.

Schädigungsparameter. Ein Überblick über die bedeutendsten Schädigungsparameter von Patienten in der Physio- und Ergotherapie innerhalb der Neurorehabilitation wurde von Langhorne und Kollegen [31] für Patienten nach Schlaganfall beschrieben (Tab. 1). Für diese unterschiedlichen Schädigungsparameter existieren auch verschiedene Assessments in der Neurorehabilitation (Tab. 2 ; [31]).

\section{Körperfunktion}

\section{Das Bewegungsausmaß}

Goniometer. Die Messung der Gelenkwinkel wird sehr häufig mit einem Goniometer durchgeführt. Vor allem an mittleren und kleinen Gelenken weist das Goniometer eine gute Reliabilität und Validität auf $[3,18,47]$. An großen Gelenken, wie Schulter oder Hüftgelenk, ist die Messung mit dem Goniometer hinsichtlich der Zuverlässigkeit zwischen mehreren Messungen des gleichen Untersuchers (Intrarater-Reliabilität) häufig gut, jedoch bei unterschiedlichen Testern (Interrater-Reliabilität) ungenügend [13, 24, 23]. Beispielsweise treten am Schultergelenk Unterschiede von $20^{\circ}$ bis $25^{\circ}$ pro Messung durch einen Wechsel des

\begin{tabular}{|c|c|}
\hline ICF-Ebene & Schädigungsparameter \\
\hline \multirow{9}{*}{$\begin{array}{l}\text { Körper- } \\
\text { struktur } \\
\text { und } \\
\text { Funktion }\end{array}$} & Gehirn \\
\hline & kardiovaskuläres System \\
\hline & $\begin{array}{l}\text { Störungen des Bewusstseins } \\
\text { und der Aufmerksamkeit }\end{array}$ \\
\hline & Arm, Schulter und Bein \\
\hline & einzelne Gehbewegungen \\
\hline & $\begin{array}{l}\text { Mobilität und Stabilität der } \\
\text { Gelenke }\end{array}$ \\
\hline & $\begin{array}{l}\text { Muskelkraft, Muskelspannung, } \\
\text { Reflexe }\end{array}$ \\
\hline & Kraftausdauer \\
\hline & $\begin{array}{l}\text { Bewegungskontrolle/Koordi- } \\
\text { nation }\end{array}$ \\
\hline \multirow[t]{12}{*}{ Aktivität } & Positionen ändern \\
\hline & gehen \\
\hline & Toilettengang \\
\hline & anziehen \\
\hline & umhergehen und -fahren \\
\hline & Körper waschen \\
\hline & Hand- und Armgebrauch \\
\hline & essen und trinken \\
\hline & Nahrung zubereiten \\
\hline & Transportmittel nutzen \\
\hline & entspannen \\
\hline & Hausarbeiten durchführen \\
\hline \multirow[t]{6}{*}{ Teilhabe } & einkaufen \\
\hline & arbeiten \\
\hline & aufräumen \\
\hline & kochen \\
\hline & Hobbys nachgehen \\
\hline & Familie/Freunde treffen \\
\hline
\end{tabular}

Testers auf [13]. Gleiches gilt auch für die Messung am Hüftgelenk [24]. Bei der Messung der Hüftabduktion und -innenrotation treten sogar bedeutende Unterschiede beim gleichen Untersucher auf [24].

\section{Im Therapiealltag kann die Messung} der Gelenkwinkel mit dem Goniometer an kleinen und mittleren Gelenken zuverlässig durchgeführt werden. Für Schulter und Hüftgelenk kann dieses Messverfahren ebenfalls verwendet werden, wenn der gleiche Tester die Untersuchung z. B. vor und direkt nach der Behandlung durchführt. Dies gilt jedoch nicht für Hüftabduktion und -innenrotation. 
Inklinometer. Ein Beispiel dafür ist die Therapie und Messung der Schulterabduktion und -außenrotation bei eingeschränkter Beweglichkeit der paretischen Seite nach Schlaganfall. Hier kann mit dem Goniometer zuverlässig die Beweglichkeit der Schulter vor und direkt nach der Behandlung, z. B. nach einer Dehnlagerung, vom gleichen Therapeuten gemessen werden, um den Erfolg der Therapie objektiv abzubilden.

Die Goniometermessung an Schulterund Hüftgelenk eignet sich nicht, wenn innerhalb der Rehabilitation oder im Praxisalltag mehrere unterschiedliche Tester die Messung durchführen müssen. Dieser Fall kann durch Urlaub, Krankheit oder Stationsverlegungen jedoch eintreten. Daher sollte für die Gelenkmessung an Schulter und Hüfte ein Inklinometer verwendet werden. Dieses weist laut der Untersuchung von Herrero und Kollegen bei der Messung des Hüftgelenks an Patienten mit spastischer Tetraparese eine sehr gute Intra- und Interrater-Reliabilität auf [24]. Auch an kleinen Gelenken misst das Inklinometer Veränderungen genauso zuverlässig wie ein Goniometer.

\section{Ein Wechsel des Testinstrumentes bei der Untersuchung des gleichen Gelenks ist nicht zu empfehlen, da Goniometer und Inklinometer Messunterschiede von $2^{\circ}$ bis $20^{\circ}$ aufweisen [30]. Die Therapeuten müssen sich daher vor der Messung entscheiden, welche Messvariante sie bevorzugen, und diese dann kontinuierlich beibehalten.}

\section{Die Kraft}

Medical Research Council. Um den Kraftgrad einzelner Muskeln zu überprüfen, eignet sich die Medical Research Council (MRC). Diese Skala teilt die Kraft von null Punkte bis fünf Punkte ein (Tab. 3).

Die Testung erfolgt hierbei manuell [50]. In einer Untersuchung von Kleyweg und Kollegen [29] konnte bei beatmeten Patienten mit Guillain-Barré-Syndrom eine sehr hohe Zuverlässigkeit für die manuelle Krafttestung mit der MRC an sechs verschiedenen Muskelgruppen im Sitz ermittelt werden $\left(\mathrm{K}=0,85, \mathrm{r}^{2}=0,96\right)$. Hierbei wurden die Muskelgruppen für die Dorsalextension der Hand, die Ellenbogenflexion und die Schulterabduktion sowie die Hüftflexion,

\begin{tabular}{|c|c|c|}
\hline ICF-Ebene & Messung & Test \\
\hline \multirow{10}{*}{$\begin{array}{l}\text { Körperstruktur } \\
\text { und Funktion }\end{array}$} & Bewegungsausmaß & Goniometer, digitales Inklinometer \\
\hline & Kraft & manueller Krafttest, Kraftdynamometrie \\
\hline & Bewusstsein & Glasgow Coma Scale \\
\hline & Aufmerksamkeit/Neglect & Bells-and-Star-Cancellation-Tests \\
\hline & Spastik & $\begin{array}{l}\text { Modified Ashworth Scale, Modified Tardieu } \\
\text { Scale }\end{array}$ \\
\hline & Sensorik & Nottingham Sensory Assessment \\
\hline & Schmerz & Visuelle Analogskala, Ritchie Articular Index \\
\hline & Ausdauer & $\begin{array}{l}\text { 6-Minuten-Gehtest, } \\
\text { Five Times Sit-to-Stand Test }\end{array}$ \\
\hline & Ataxie & Scale for the Assessment and Rating of Ataxia \\
\hline & Apraxie & Test for Upper Limb Apraxia \\
\hline \multirow[t]{14}{*}{ Aktivität } & Gehfähigkeit & Functional Ambulation Categories \\
\hline & Aufstehen und Gehen & Timed-up-and-go-Test \\
\hline & Sitzen, Aufstehen, Stehen & Berg Balance Scale \\
\hline & Gangstörung/sicheres Gehen & Dynamic Gait Index \\
\hline & sicheres Stehen & Functional-Reach-Test \\
\hline & $\begin{array}{l}\text { Fähigkeit, sich aufzusetzen und } \\
\text { zu sitzen }\end{array}$ & Trunk-Control-Test \\
\hline & allgemeine Aktivitäten & Functional Independent Measure \\
\hline & $\begin{array}{l}\text { allgemeine Mobilität und } \\
\text { Armaktivitäten }\end{array}$ & Motor Assessment Scale \\
\hline & allgemeine Mobilität & Rivermead Mobility Index \\
\hline & grobmotorisches Greifen & Box-and-Block-Test \\
\hline & feinmotorisches Greifen & Nine-Hole-Peg-Test \\
\hline & allgemeine Armaktivitäten & $\begin{array}{l}\text { Wolf-Motor-Function-Test, Jebsen-Taylor- } \\
\text { Hand-Test }\end{array}$ \\
\hline & $\begin{array}{l}\text { Schrittlänge und Gehge- } \\
\text { schwindigkeit }\end{array}$ & 10-Meter-Gehtest \\
\hline & Gehleistung & 6-Minuten-Gehtest \\
\hline \multirow[t]{5}{*}{ Teilhabe } & $\begin{array}{l}\text { Integration in das soziale } \\
\text { Umfeld }\end{array}$ & Reintegration to Normal Living Index \\
\hline & Lebensqualität & EuroQuol-5 Dimensions \\
\hline & $\begin{array}{l}\text { Nutzung der oberen Extremität } \\
\text { im Alltag }\end{array}$ & Motor Activity Log \\
\hline & $\begin{array}{l}\text { Mobilität, Lebensqualität, } \\
\text { Ressourcen im Alltag }\end{array}$ & Stroke Impact Scale \\
\hline & $\begin{array}{l}\text { Alltag, Mobilität und Hausar- } \\
\text { beit }\end{array}$ & Frenchay Activities Index \\
\hline
\end{tabular}

Tab. 3 Krafteinteilung nach der Medical Research Council (MRC)

Kraftstufe Definition

\begin{tabular}{|c|c|}
\hline 0 & Der Muskel ist absolut inaktiv. \\
\hline 1 & $\begin{array}{l}\text { Eine Kontraktion der Muskulatur oder ein Anspannen der Sehne wird deutlich } \\
\text { vom Tester vernommen. }\end{array}$ \\
\hline 2 & Die Bewegung wird vollständig ohne Schwerkrafteinfluss geführt. \\
\hline 3 & Die Bewegung wird vollständig gegen die Schwerkraft geführt. \\
\hline 4 & $\begin{array}{l}\text { Die Bewegung wird vollständig gegen die Schwerkraft mit einem starken } \\
\text { Widerstand geführt. }\end{array}$ \\
\hline 5 & $\begin{array}{l}\text { Die Bewegung wird vollständig gegen die Schwerkraft mit maximalem Wider- } \\
\text { stand geführt. }\end{array}$ \\
\hline
\end{tabular}




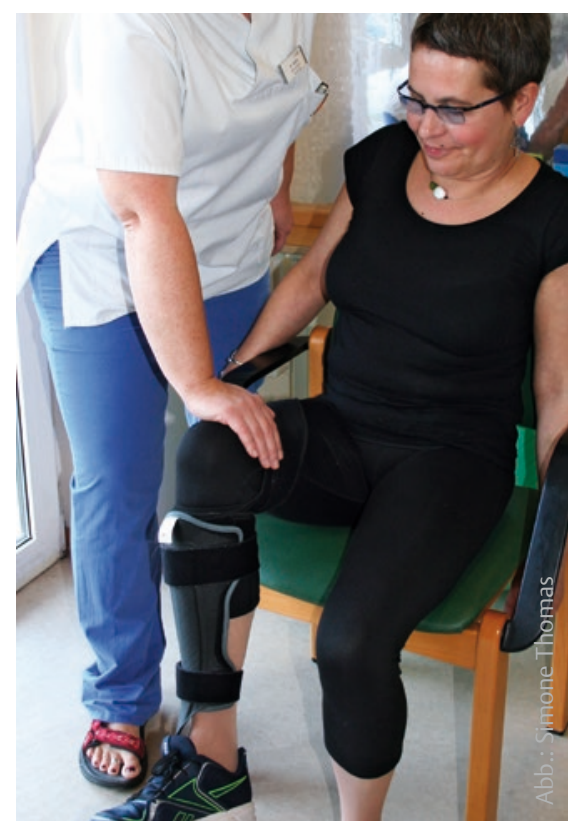

Abb. 1 MRC Stufe 5 für Hüftflexion

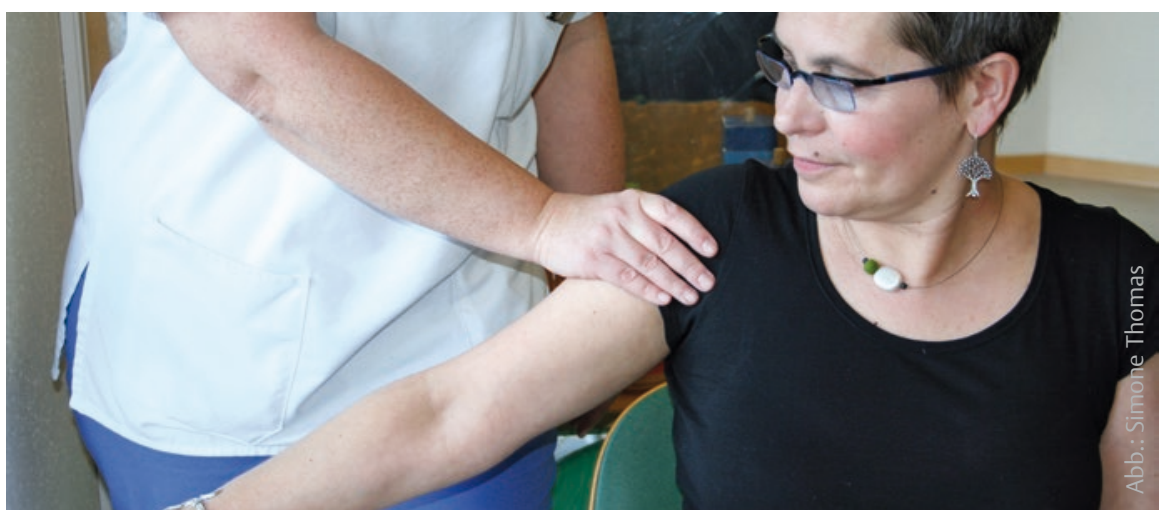

Abb. 3 MRC Stufe 4 für Schulterabduktion

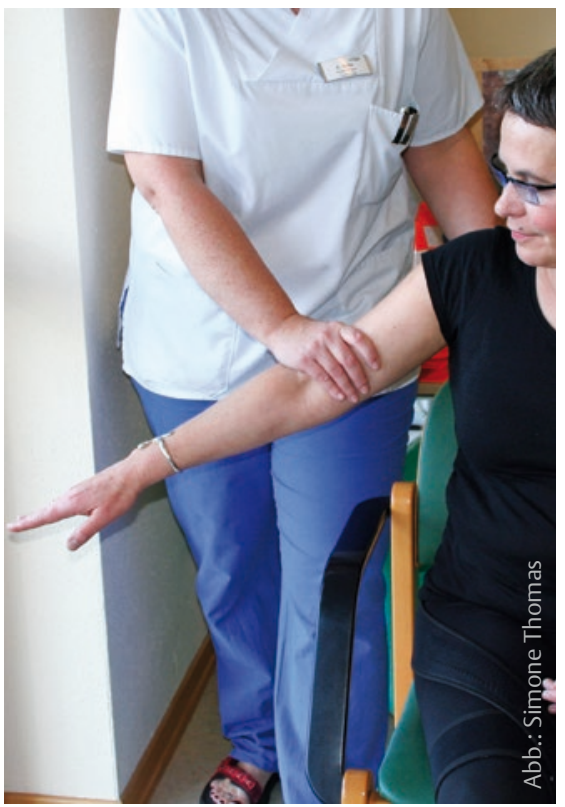

Abb. 4 MRC Stufe 5 für Schulterabduktion

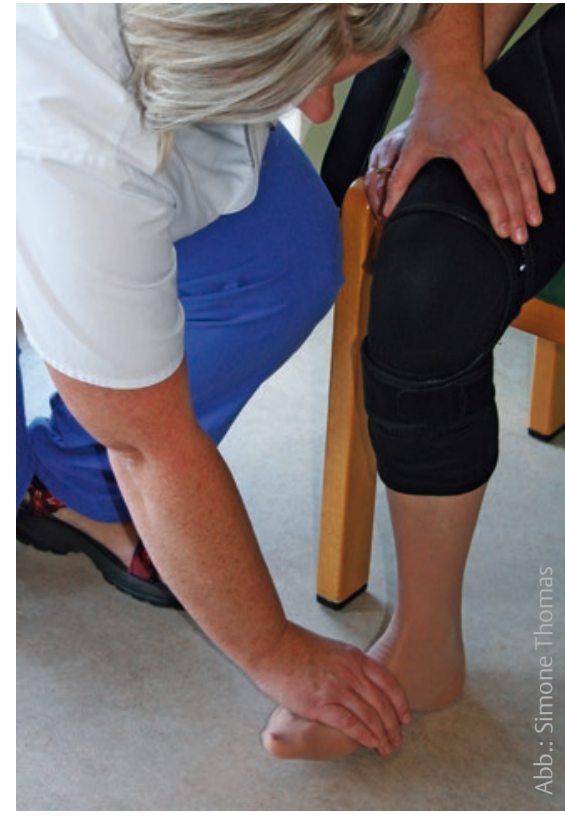

Abb. 2 MRC Stufe 5 Dorsalextension Fuß

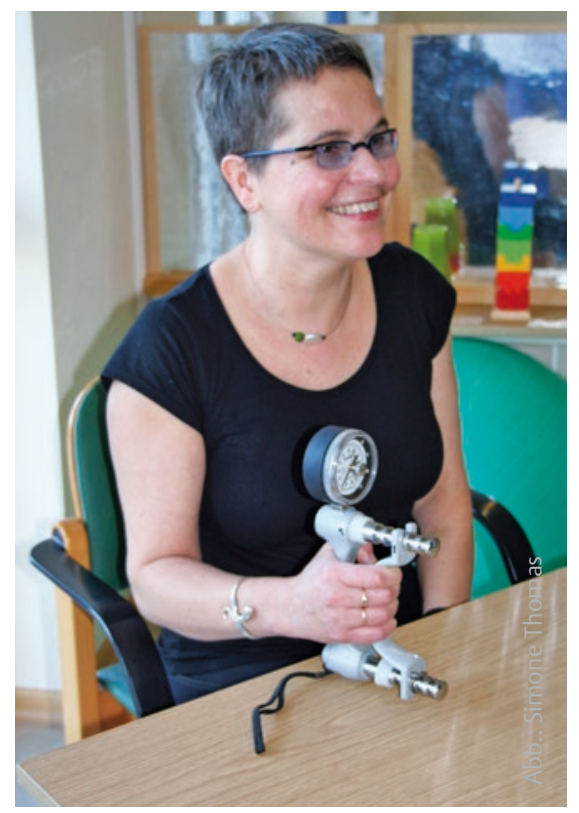

Abb. 5 Griffkraft-Dynamometrie
Tab. 4 Bewertungsskala des Motricity Index (freie deutsche Übersetzung nach [11]) Punkte Pinzettengriff (Würfel ca. 2,5 cm)

\begin{tabular}{|c|c|}
\hline 0 & keine Aktivität \\
\hline 11 & $\begin{array}{l}\text { jegliche Bewegung, die einem } \\
\text { Greifen nahekommt }\end{array}$ \\
\hline 19 & $\begin{array}{l}\text { kann Würfel ergreifen, aber nicht } \\
\text { gegen die Schwerkraft halten }\end{array}$ \\
\hline 22 & $\begin{array}{l}\text { kann Würfel gegen die Schwer- } \\
\text { kraft halten, aber nicht gegen } \\
\text { leichten Zug }\end{array}$ \\
\hline 26 & $\begin{array}{l}\text { kann Würfel gegen leichten Zug } \\
\text { halten, aber nicht gegen starken } \\
\text { Zug }\end{array}$ \\
\hline 33 & $\begin{array}{l}\text { kann Würfel gegen starken Zug } \\
\text { halten }\end{array}$ \\
\hline & Arm und Bein \\
\hline 0 & absolut inaktiv \\
\hline 9 & $\begin{array}{l}\text { Kontraktion der Muskulatur } \\
\text { sichtbar }\end{array}$ \\
\hline 14 & $\begin{array}{l}\text { Bewegung möglich, aber ohne } \\
\text { vollständiges Bewegen gegen } \\
\text { Schwerkrafteinfluss }\end{array}$ \\
\hline 19 & $\begin{array}{l}\text { vollständige Bewegung gegen die } \\
\text { Schwerkraft möglich }\end{array}$ \\
\hline 25 & $\begin{array}{l}\text { Bewegung gegen die Schwerkraft } \\
\text { mit Widerstand möglich, aber } \\
\text { weniger als auf der anderen Seite }\end{array}$ \\
\hline 33 & normale Muskelkraft \\
\hline
\end{tabular}

Knieextension und Dorsalextension des oberen Sprunggelenks im Sitz getestet (Abb. 1 u. Abb. 2) [29].

Um den Widerstand für Stufe 4 und $5 \mathrm{zu}$ objektivieren, bietet es sich an, diesen mit gleicher Kraft, aber an unterschiedlichen Punkten zu setzen. Für Kraftstufe 4 kann die Hand des Untersuchers zwei Finger breit unter dem zu testenden Gelenk und für Stufe 5 über dem distal angrenzenden Gelenk platziert werden (Abb. 3 u. Abb. 4).

Weiterhin ist es möglich, diverse Dynamometer zur Erfassung der Kraft einzusetzen [45]. Diese können sehr einfach vom Therapeuten bedient werden und zeigen die Kraft in Kilogramm oder Pfund analog oder digital an. Zu nennen sind hierbei das GriffkraftDynamometer für Hand- und Fingerflexoren (Abb. 5) und das Handheld-Dynamometer für die obere und untere Extremität.

Motricity Index. Bei Patienten nach einem Schlaganfall eignet sich besonders der Motricity Index, um die Kraft zu testen (Tab. 4) [11]. Die Kraftmessung für die Schulter- 


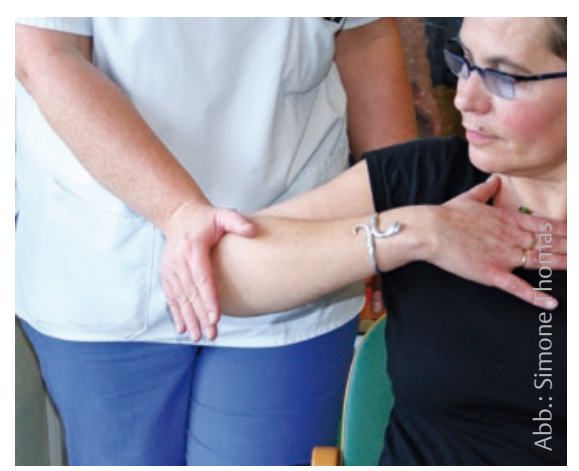

Abb. 6 Motricity Index Schulterabduktion Stufe 5

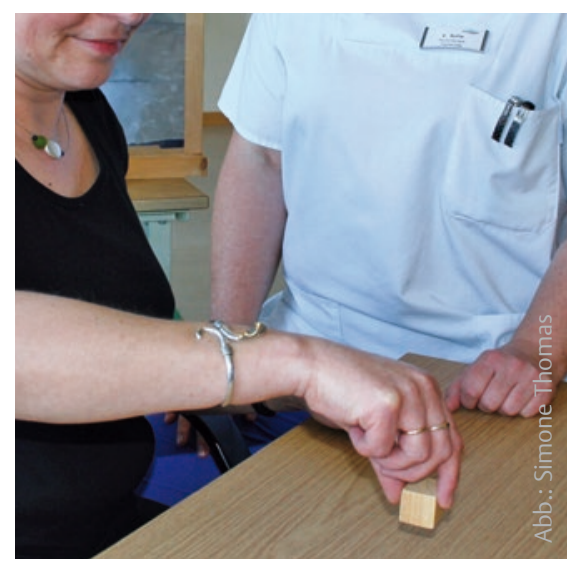

Abb. 7 Motricity Index Würfel anheben

abduktion wird hier mit der Hand auf der Brust und für Ellenbogenbeugung aus einer $90^{\circ}$ flektierten Position durchgeführt, wodurch vorhandene Beugekontraktoren oder Spastik an Schulter und Ellenbogen berücksichtigt werden (Abb. 6).

Weiterhin wird statt der Kraft der Dorsalextensoren des Handgelenks wie bei der MRC die feinmotorische Greiffunktion überprüft. Es soll dafür ein $2,5 \mathrm{~cm}$ großer Würfel zwischen Daumen und Zeigefinger angehoben und festgehalten werden (Abb. 7). Die Untersuchung der unteren und oberen Extremität wird, wie auch bei der MRC, komplett im Sitz mit Fußbodenkontakt durchgeführt. Die Skalierung des Tests ist in Tab. 4 dargestellt. Dieser Test kann v.a. bei sehr schweren Paresen der oberen Extremität, wo Messungen von Greif- oder Halteaktivitäten nicht möglich sind, Aufschluss über den motorischen Verlauf geben.

\section{Das Bewusstsein einschätzen}

Das Bewusstsein von Patienten nach Schädel-Hirn-Verletzungen wird international mit der Glasgow Coma Scale (GCS) beurteilt [56]. Diese Skala prüft Augenöffnung, Motorik und Sprache des Patienten (Tab. 5).
Tab. 5 Glasgow-Koma-Skala (freie deutsche Übersetzung nach [56])

\begin{tabular}{|l|l|l|}
\hline Augen öffnen & spontan & 4 \\
\hline & nach Aufforderung & 3 \\
\hline newegung & keine Reaktion & 1 \\
\hline & normal & 6 \\
\hline & $\begin{array}{l}\text { gezielte Abwehrre- } \\
\text { aktion }\end{array}$ & 5 \\
\hline & $\begin{array}{l}\text { ungezielte Abwehr- } \\
\text { reaktion }\end{array}$ & 4 \\
\hline & Beugebewegungen & 3 \\
\hline & Streckbewegungen & 2 \\
\hline keine Reaktion & 1 \\
\hline Sprache & orientiert & 5 \\
\hline & verwirrt & 4 \\
\hline & einzelne Worte & 3 \\
\hline & Laute & 2 \\
\hline & keine Reaktion & 1 \\
\hline
\end{tabular}

Ein niedriger Punktwert auf der GCS zu Beginn der Erkrankung korreliert laut einer Untersuchung von Balestreri und Kollegen mit einem niedrigen Wert auf der Glasgow Outcome Scale, einer Skala zur Beurteilung der Selbstständigkeit außerhalb der Klinik [4]. Bei einer Gesamtpunktzahl von 8 oder weniger liegt ein schweres Schädel-Hirn-Trauma mit deutlich negativen Auswirkungen auf die Selbstständigkeit und Selbstversorgung in den nächsten fünf Jahren vor [4].

\section{Spastik eindeutig feststellen}

Ein häufig eingesetztes Verfahren zur Bestimmung der Spastik ist die Messung des Muskelwiderstands während passiver Bewegung mit der Ashworth-Skala bzw. mit der modifizierten Ashworth-Skala. Jedoch ist nicht jeder Muskelwiderstand gleich eine Spastik. Weiterhin beeinflussen Kontrakturen die Messungen mit der Ashworth-Skala deutlich [39]. Die Interrater- und auch die Intrarater-Reliabilität bei Patienten mit spastischer Lähmung ist moderat [2, 21, 40, 46].

Eine Alternative zur Ashworth-Skala stellt die modifizierte Tardieu-Skala dar $[21,55]$. Sie kann im Gegensatz zur Ashworth-Skala eine Spastik von z.B. einer reinen Kontraktur oder Muskelverspannung zuverlässig unterscheiden [21, 55]. Außerdem weist sie im Vergleich zur AshworthSkala eine etwas höhere Reliabilität auf [40]. Die modifizierte Tardieu-Skala misst zwei verschiedene Parameter:

\begin{tabular}{|l|l|}
\hline Tab. 6 & Punkte Tardieu-Skala nach [42] \\
\hline Stufe & Beschreibung \\
\hline 0 & $\begin{array}{l}\text { kein Widerstand während der } \\
\text { passiven Bewegung durch das volle } \\
\text { Bewegungsausmaß }\end{array}$ \\
\hline 1 & $\begin{array}{l}\text { leichter Widerstand während } \\
\text { der passiven Bewegung ohne } \\
\text { klaren Stopp in einer bestimmten } \\
\text { Winkelstellung }\end{array}$ \\
\hline 2 & $\begin{array}{l}\text { klarer Stopp in einer bestimmten } \\
\text { Winkelstellung, der die passive } \\
\text { Bewegung unterbricht, aber dann } \\
\text { nachlässt }\end{array}$ \\
\hline 3 & $\begin{array}{l}\text { erschöpflicher Klonus in einer } \\
\text { bestimmten Winkelstellung kürzer } \\
\text { als 10 Sekunden, wenn die Position } \\
\text { gehalten wird }\end{array}$ \\
\hline 4 & $\begin{array}{l}\text { unerschöpflicher Klonus in einer } \\
\text { bestimmten Winkelstellung länger } \\
\text { als 10 Sekunden, wenn die Position } \\
\text { gehalten wird }\end{array}$ \\
\hline 3
\end{tabular}

\section{- Bewegungsausmaß}

- Bewegungsqualität

Die Durchführung der rein passiven Testbewegungen erfolgt in der modifizierten Version mit zwei statt mit ursprünglich drei verschiedenen Bewegungsgeschwindigkeiten, immer zum gleichen Tageszeitpunkt in Rückenlage mit, wenn möglich, ausgestreckten Extremitäten [34, 40].

Die Bewegungsgeschwindigkeiten werden wie folgend definiert:

- V1 = langsames Bewegen (komplettes Bewegungsausmaß)

- V3 = sehr schnelles Bewegen (schneller als Fallen mit der Schwerkraft)

Um das Bewegungsausmaß einzuschätzen, wird der Gelenkwinkel nach V1 und nochmals direkt nach V3 gemessen. Die Differenz der Gelenkwinkel (V1-V3) wird notiert. Zusätzlich erfolgt die Beurteilung der Bewegungsqualität für V3 in Punkten (Tab. 6.)

\section{Aktivität}

Das Gehen bewerten

Die Fähigkeit, zu gehen, kann sehr zuverlässig mithilfe der Functional Ambulation Categories (FAC) ermittelt werden (Tab. 7) [26].

Gehfähigkeit. Um diese einschätzen zu können, muss der Patient auf einer Gehstrecke mit einer Länge von 10 oder $15 \mathrm{~m}$ unabhängig vom Hilfsmittel bei größtmöglicher Selbstständigkeit gehen [1, 41]. Die Länge der Gehstrecke ist bei jeder Messung gleich 
zu wählen und sollte auf dem Dokumentationsblatt vermerkt werden. Hilfsmittel können vom Patienten verwendet werden $[1,41]$.

Schlüsselfragen. Weiterhin beschreiben Mehrholz und Arbeitsgruppe Schlüsselfragen, die hilfreich sind, um die FAC sicher einschätzen zu können [41]. Gerade die Unterscheidung von FAC1 oder FAC2 (Tab. 7) fällt nach eigener Erfahrung schwer. Folgende Fragen in dem Artikel von Mehrholz und Arbeitsgruppe sind zur Unterscheidung von FAC1 und FAC2 aufgeführt:

\begin{tabular}{|l|l|}
\hline $\begin{array}{l}\text { Tab. } 7 \\
\text { (Übersetzung nach [41]) }\end{array}$ \\
\hline FAC & Definition \\
\hline 0 & $\begin{array}{l}\text { Der Patient läuft im oder am Barren } \\
\text { oder benötigt zwei Personen zur } \\
\text { physischen Unterstützung beim } \\
\text { Gehen auf ebenem Untergrund. }\end{array}$ \\
\hline 1 & $\begin{array}{l}\text { Der Patient benötigt eine Person zur } \\
\text { physischen Unterstützung auf ebenem } \\
\text { Untergrund. }\end{array}$ \\
\hline 2 & $\begin{array}{l}\text { Der Patient benötigt eine Person zur } \\
\text { kontinuierlichen oder diskontinuier- } \\
\text { lichen Sicherung des Gleichgewichts } \\
\text { oder der Koordination. }\end{array}$ \\
\hline 3 & $\begin{array}{l}\text { Der Patient benötigt eine Person zur } \\
\text { Begleitung auf ebenem Untergrund. }\end{array}$ \\
\hline 4 & $\begin{array}{l}\text { Der Patient benötigt eine Person zur } \\
\text { Sicherung des Ganges bei Uneben- } \\
\text { heiten oder an der Treppe. }\end{array}$ \\
\hline 5 & $\begin{array}{l}\text { Der Patient ist vollständig selbst- } \\
\text { ständig gehfähig. }\end{array}$ \\
\hline
\end{tabular}

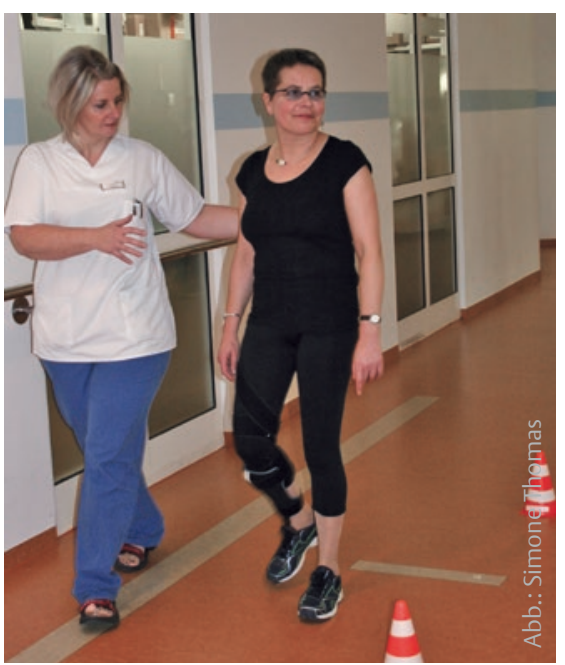

Abb. 10 Gehen mit horizontalen Kopfbewegungen (links)
- Ist es deutlich sichtbar, dass der Tester dem Patienten Gewicht abnimmt?

- War es mindestens ein Mal notwendig, dass der Tester das Bein des Patienten bewegt?

- Ist es deutlich sichtbar, dass der Tester den Patienten am Brustkorb unterstützt? Werden mindestens zwei Fragen mit „Ja“ beantwortet, liegt FAC 1 vor. Ist die Antwort auf mindestens zwei Fragen „Nein“, liegt mindestens FAC2 vor [41].

Gleichgewicht. Neben der Gehfähigkeit kann auch das Gleichgewicht im Gang mit dem Dynamic Gait Index (DGI) bewertet werden [53]. Es hat sich in der klinischen Praxis bewährt, diesen Test ab FAC2 bei diskontinuierlicher Gleichgewichtssicherung im Gang

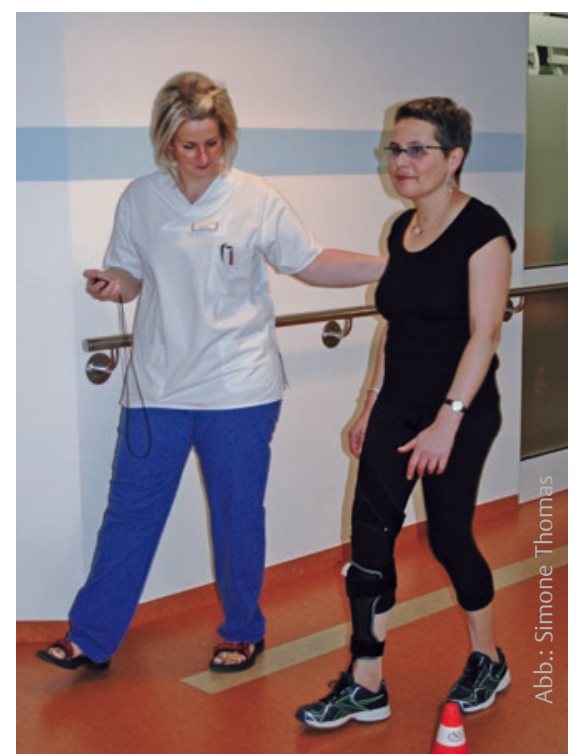

Abb. 8 Gehen mit normaler Geschwindigkeit

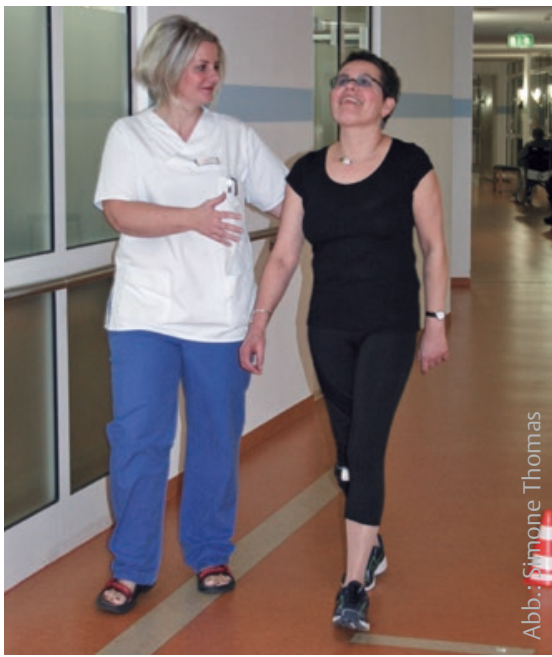

Abb. 11 Gehen mit vertikalen Kopfbewegungen (oben) durchzuführen. Hierbei wird auf einer Ebene von ca. $6 \mathrm{~m}$ (20 Fuß) das Gehen in acht verschiedenen Varianten mit jeweils 0 bis 3 Punkten bewertet (Abb. 8, Abb. 9, Abb. 10, Abb. 11 u. Abb. 12) [51, 52].

In einigen deutschen Übersetzungen soll für die Beurteilung des Gleichgewichts im Gang eine Gehstrecke von $20 \mathrm{~m}$ absolviert werden. Der englische DGI sieht für die Länge der Testbahn jedoch nur 20 Fuß (rund $6 \mathrm{~m}$ ) vor. Für die lange Gehstrecke von 20 m liegen, im Gegensatz zur 6-mGehstrecke, bislang jedoch noch keine Daten zu den psychometrischen Eigenschaften des Tests vor. Daher wird aus eigenem Ermessen empfohlen, den Test mit der originalen Gehstrecke von rund $6 \mathrm{~m}$ durchzuführen. Vor allem in Praxisräumen oder

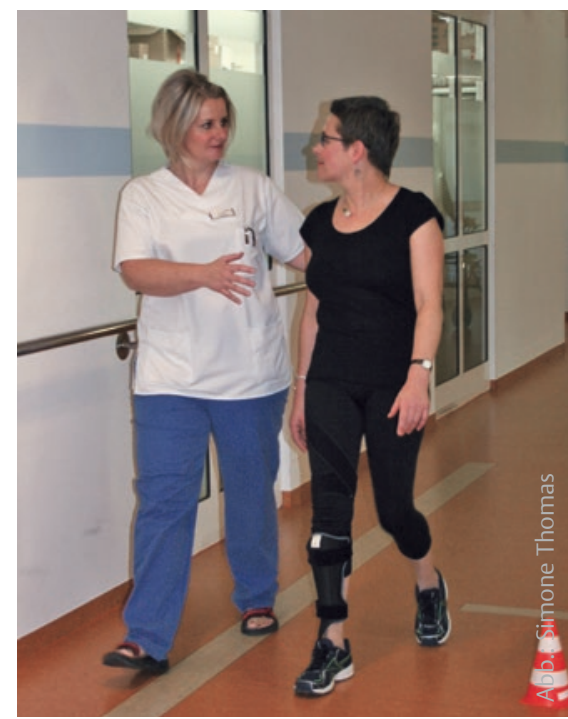

Abb. 9 Gehen mit horizontalen Kopfbewegungen (rechts)

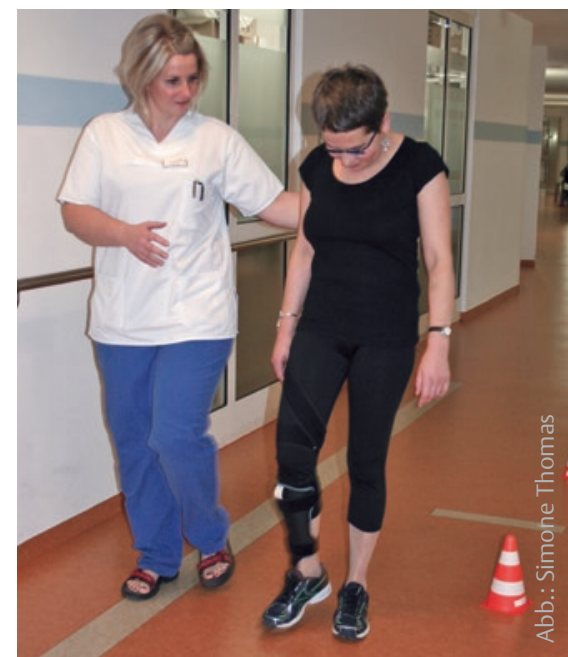

Abb. 12 Gehen mit vertikalen Kopfbewegungen (unten) 


\begin{tabular}{|c|c|}
\hline Aufgabe / Punkte & Inhalt \\
\hline 1. Geradeaus gehen & $\begin{array}{l}\text { Gehen Sie in normalem Tempo bis zur markierten Stelle } \\
\text { ( } 20 \text { Fuß/6 m). }\end{array}$ \\
\hline 3 & $\begin{array}{l}\text { normale Geschwindigkeit, keine Hilfsmittel, normales } \\
\text { Gangbild, kein Schwanken }\end{array}$ \\
\hline 2 & $\begin{array}{l}\text { benutzt Hilfsmittel, Geschwindigkeit verlangsamt, leichtes } \\
\text { Schwanken }\end{array}$ \\
\hline 1 & $\begin{array}{l}\text { Geschwindigkeit sehr langsam, deutliche Abweichungen } \\
\text { vom normalen Gangmuster, deutliches Schwanken }\end{array}$ \\
\hline 0 & 20 Fuß/6 m Gehen nur mit Hilfe möglich \\
\hline $\begin{array}{l}\text { 2. Gehen mit wechselnder } \\
\text { Geschwindigkeit }\end{array}$ & $\begin{array}{l}\text { Gehen Sie in normalem Tempo. } \\
\text { Wenn ich sage „schnell“, gehen Sie sofort schnell (5 } \\
\text { Fuß/1,5 m). } \\
\text { Wenn ich sage „ruhig“, gehen Sie sofort langsam ( } 5 \\
\text { Fuß/1,5 m). }\end{array}$ \\
\hline 3 & $\begin{array}{l}\text { Änderung der Geschwindigkeit deutlich erkennbar, kein } \\
\text { Schwanken }\end{array}$ \\
\hline 2 & $\begin{array}{l}\text { benutzt Hilfsmittel, nur leichte Änderung der Geschwin- } \\
\text { digkeit erkennbar, leichtes Schwanken, Gangabweichungen }\end{array}$ \\
\hline 1 & $\begin{array}{l}\text { kaum Änderung der Geschwindigkeit erkennbar, starke } \\
\text { Gangabweichung, Stolpern, ohne zu fallen }\end{array}$ \\
\hline 0 & $\begin{array}{l}\text { keine Veränderung der Gehgeschwindigkeit sichtbar, } \\
\text { Stolpern mit Fallneigung, greift nach der Wand }\end{array}$ \\
\hline $\begin{array}{l}\text { 3. Gehen mit horizontalen } \\
\text { Kopfbewegungen }\end{array}$ & $\begin{array}{l}\text { Laufen Sie geradeaus bis zur Markierung ( } 20 \text { Fuß/6m). } \\
\text { Wenn ich sage „links“, dann drehen Sie den Kopf so zügig } \\
\text { wie möglich während des Gehens nach links, halten Sie ihn } \\
\text { dort ( } 5 \text { Fuß/1,5 m nach Start). } \\
\text { Wenn ich sage „rechts“, tun Sie dasselbe zur rechten Seite } \\
\text { (10 Fuß/3 m nach Start). } \\
\text { Wenn ich sage „vor“, blicken Sie nach vorn (15 Fuß/4,5 m } \\
\text { nach Start). } \\
\text { Gehen Sie während der Aufgaben immer geradeaus weiter, } \\
\text { ohne anzuhalten. }\end{array}$ \\
\hline 3 & flüssige Kopfbewegung, kein Schwanken \\
\hline 2 & $\begin{array}{l}\text { leichtes Schwanken, Veränderung der Spurbreite, Nutzen } \\
\text { eines Hilfsmittels }\end{array}$ \\
\hline 1 & verlangsamte Bewegungen, Stolpern, ohne zu fallen \\
\hline 0 & starkes Stolpern, Stehenbleiben, Festhalten an der Wand \\
\hline $\begin{array}{l}\text { 4. Gehen mit vertikalen } \\
\text { Kopfbewegungen }\end{array}$ & $\begin{array}{l}\text { Laufen Sie geradeaus bis zur Markierung (6 m). } \\
\text { Wenn ich sage „oben“, heben Sie den Kopf so zügig wie } \\
\text { möglich während des Gehens nach oben, halten Sie ihn } \\
\text { dort (ab 1,5 m nach Start). } \\
\text { Wenn ich sage „unten“, tun Sie dasselbe nach unten (ab } \\
3 \text { m nach Start). } \\
\text { Wenn ich sage „vor“, blicken Sie nach vorn (ab 4,5 m). } \\
\text { Gehen Sie während der Aufgaben immer geradeaus weiter, } \\
\text { ohne anzuhalten. }\end{array}$ \\
\hline 3 & flüssige Kopfbewegung, kein Schwanken \\
\hline 2 & $\begin{array}{l}\text { leichtes Schwanken, Veränderung der Spurbreite, Nutzen } \\
\text { eines Hilfsmittels }\end{array}$ \\
\hline 1 & verlangsamte Bewegungen, Stolpern, ohne zu fallen \\
\hline 0 & starkes Stolpern, Stehenbleiben, Festhalten an der Wand \\
\hline
\end{tabular}

Stationsgängen ist die 6-m-Gehstrecke im Gegensatz zu der Variante mit $20 \mathrm{~m}$ besser implementierbar.

Weiterhin existiert eine sehr zuverlässige Kurzversion des DGI mit vier statt acht Gangvariationen. Dadurch wird der Test noch praktikabler und lässt sich schnell im Therapiealltag durchführen (Tab. 8) [33].

Um das normale Gangtempo während Aufgabe 1 einschätzen zu können, empfiehlt es sich, mit einer Stoppuhr die benötigte Zeit für das Gehen von $6 \mathrm{~m}$ zu messen. Der Normwert für die Gehgeschwindigkeit von älteren gesunden Probanden beträgt 0,9 $\mathrm{m} / \mathrm{s}$ [48]. Die normale Gehdauer für $6 \mathrm{~m}$ liegt demnach bei rund $7 \mathrm{~s}$.

\section{Armfunktionen einschätzen}

Wolf-Motor-Function-Test und Jebsen-Taylor-Hand-Test. Beide sind sehr bekannte und beliebte Messinstrumente für die obere Extremität [45]. Mit diesen Tests können eine Reihe von grob- und feinmotorischen Armaktivitäten gemessen werden. Jedoch benötigt man für diese Tests Zeit (mindestens $30 \mathrm{~min}$ ) und z. T. Videoaufnahmen oder mehrtägige Schulungen der Untersucher. Außerdem sind Fähigkeiten und Aktivitäten des Patienten wie Lesen/Schreiben oder Stehen notwendig. Daher sind diese Tests bei einer sehr ausgewählten Patientenklientel, z. B. innerhalb einer Interventionsstudie, zuverlässig anwendbar [8, 15, 28, 32].

Wesentlich einfacher und schnell in der klinischen Praxis einsetzbar sind der Boxand-Block-Test für moderate Einschränkungen der Grobmotorik und der Nine-HolePeg-Test bei Feinmotorikstörungen.

Nine-Hole-Peg-Test. Der Nine-Hole-Peg-Test (NHP) kann bei mittleren bis leichten Armfunktionsstörungen verwendet werden. Bei diesem Test sollen 9 Stäbchen so schnell wie möglich aus einem Behälter in 9 Löcher gesteckt und anschließend wieder nacheinander in den Behälter zurückgelegt werden (Abb. 13, Abb. 14 u. Abb. 15).

Laut den Normwerten von Mathiowetz und Arbeitsgruppe [36] sowie Earhart [17] sind durchschnittlich $19 \mathrm{~s}$ für die rechte und 21 s für die linke Hand normal [17, 37].

Ein Probedurchgang für jede Hand ist direkt vor dem jeweiligen Test erlaubt. Sollte ein Stäbchen nach unten fallen, kann der Tester nach eigener Erfahrung dieses aufheben, es wieder in den Behälter zurücklegen oder bei der Rückrunde in ein Loch stecken. 


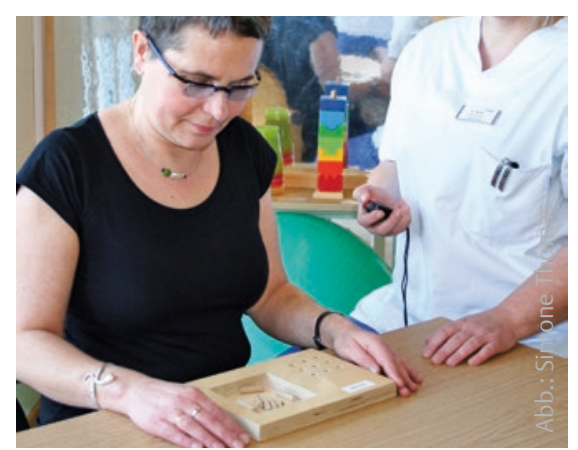

Abb. 13 Ausgangsposition

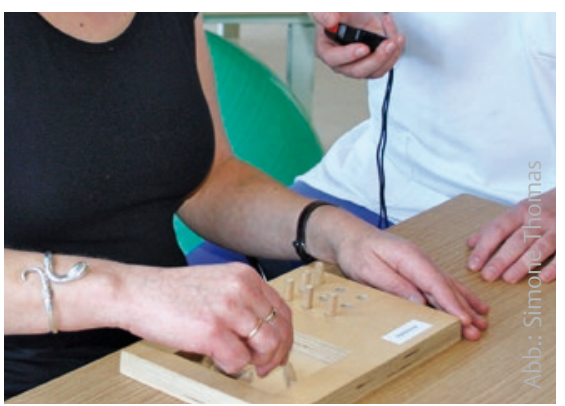

Abb. 14 Durchführung 1

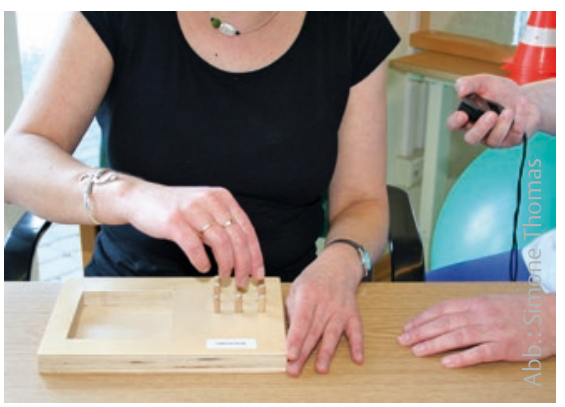

Abb. 15 Durchführung 2

Die Dauer des gesamten Durchlaufs wird mit einer Stoppuhr gemessen. Die Zeitmessung beginnt, sobald der Patient das erste Stäbchen berührt.

Eine Reduktion der Zeit um ca. 54\% bei Schlaganfallpatienten und nur $4 \%$ bei Parkinson-Patienten ohne Freezing und starken Tremor, die nicht auf Zufall beruhen kann, gilt als minimal messbare Veränderung in diesem Test $[10,17]$. Die Empfindlichkeit des Tests scheint somit abhängig von der Diagnose bzw. auch von der Schwere der Erkrankung zu sein.

In der Untersuchung von Chen [10] zur Ermittlung der minimal messbaren Veränderung nach Schlaganfall wurden Patienten mit schweren Armparesen untersucht. Der Test ist jedoch eher für Patienten mit Koordinationsstörungen der Feinmotorik entwickelt worden, wie z.B. in der Analyse zur minimal messbaren Veränderung bei Parkinson-Patienten von Earhart [17].
Die Messfehlerrate des NHP ist bei Patienten mit erheblichen Störungen der Handund Armmotorik (> als 60 s Testzeit) sehr hoch [10]. Hier sollte der Test abgebrochen und ein angepasstes Messverfahren, wie z.B. der Box-and-Block-Test oder der Motricity Index, gewählt werden.

\section{Die Interrater-Reliabilität des NHP} ist sehr gut ( $r=0,984$ rechte Hand; $r=0,993$ linke Hand) [49]. Hingegen ist die Intrarater-Reliabilität eher gering ( $r=0,459$ rechts; $r=0,442$ links) [49]. Zu beachten ist dabei, dass diese Ergebnisse nur für gesunde Probanden gelten. Die geringe Zuverlässigkeit bei Testwiederholung könnte daher durch einen schnellen Lerneffekt der gesunden Probanden erklärt werden.

Box-and-Block-Test. Ein schnelles Assessment zur Beurteilung der Grobmotorik ist der Box-and-Block-Test [36]. Dieser kann bei Patienten mit bereits vorhandenen Greiffunktionen und deutlicher Einschränkung der Feinmotorik eingesetzt werden. Der Patient sitzt laut Mathiowetz [36] während der Untersuchung vor einer Box $(\mathrm{H} / \mathrm{B} / \mathrm{T}$ $8,5 / 53,7 / 25,4 \mathrm{~cm})$ mit einer Trennwand in der Mitte (H/B/T 15,2/25,4/1 cm). Die Box schließt mit der vorderen Tischkante ab. In der einen Hälfte der Box befinden sich 150 Holzwürfel (Kantenlänge: 2,5 cm). Die kompletten Maße der Box wurden laut Mathiowetz und Arbeitsgruppe erstmals von Patricia Holser Buehler und Elizabeth Fuchs beschrieben [36]. Weiterhin beschreibt die Gruppe um Mathiowetz, dass der Untersucher dem Patienten gegenübersitzen sollte, um die Transportbewegung und die Anzahl der Steine fehlerfrei erkennen zu können.

Der Test beginnt mit der nicht betroffenen oder dominanten Hand. Die Steine befinden sich auf der Testseite. Anschließend erklärt der Untersucher dem Patienten die Durchführung des Tests [36]. Die Übersetzung der englischen Instruktion:

„Ich möchte beobachten, wie schnell Sie einen Würfel in diese Hand nehmen können (Tester zeigt auf die Testhand des Patienten) und über die Trennwand auf die andere Seite der Box befördern und fallen lassen können. Wichtig ist, dass die Fingerspitzen die Trennwand überqueren. Ich zeige es Ihnen.“

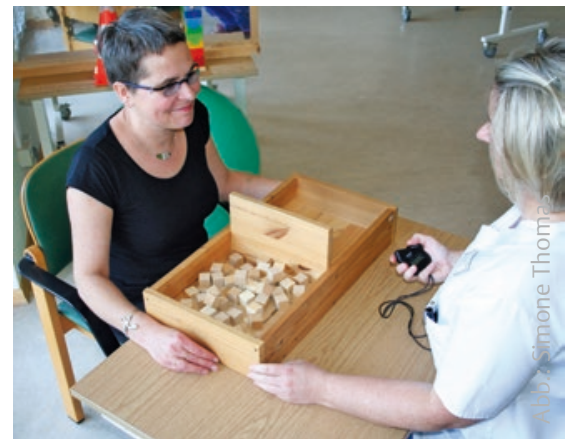

Abb. 16 Ausgangsposition

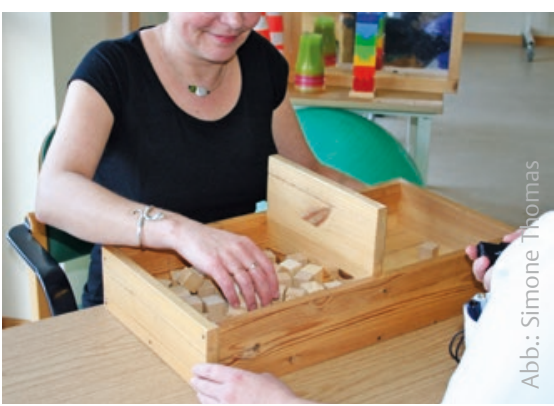

Abb. 17 Durchführung 1

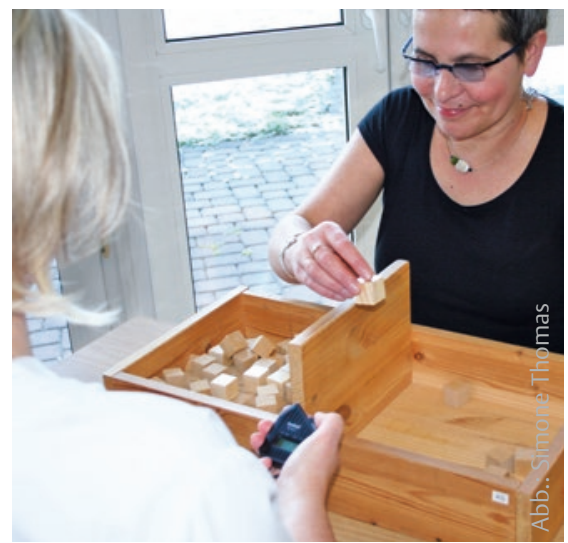

Abb. 18 Durchführung 2

Der Untersucher zeigt spiegelverkehrt das Greifen und Befördern sowie Loslassen des Würfels.

„Wenn Sie zwei oder mehrere Würfel gleichzeitig nehmen, wird nur einer gezählt. Sollte ein Würfel neben die Box fallen, nachdem Sie die Trennwand überquert haben, gilt dieser trotzdem. Versuchen Sie nicht, ihn aufzuheben. Wenn Sie den Würfel über die Wand werfen, das heißt die Wand nicht mit den Fingerspitzen überqueren, wird er nicht gezählt. Sie haben nun $15 \mathrm{~s}$ Zeit zu üben. Legen Sie beide Hände links und rechts neben die Box. Haben Sie Fragen? Wenn es losgeht, sage ich erst ,Fertig?‘ und dann ,Los! ‘.“ 
Nach dem kurzen Üben sollte der Untersucher den richtigen Test ankündigen:

„Jetzt kommt der richtige Test. Sie haben eine Minute Zeit. Danach zählen wir die Würfel. Legen Sie beide Hände links und rechts neben die Box. Fertig? [Untersucher soll 3s warten] Los! “ (Abb. 16, Abb. 17 u. Abb. 18).

Anschließend erfolgen die gleichen Anweisungen für die andere Seite [36]. Der Untersucher sollte während des Tests die ungültige Anzahl an transportierten Würfeln per Strichliste vermerken [36].

Die durchschnittliche Anzahl an Würfeln liegt, wenn der Patient unter 50 Jahre alt ist, bei ca. 80-90. Ab 50 Jahre sind zwischen 60 und 70 Würfel pro Minute unabhängig vom Geschlecht normal [5]. Die minimal bedeutende Veränderung der Würfelanzahl pro Minute liegt für Schlaganfallpatienten bei 6 Würfeln [10].

\section{Teilhabe}

Reintegration to Normal Living Index (RNLI). Er kann die mögliche Wiedereingliederung in das häusliche und soziale Umfeld erfassen $[35,59,60]$. Per Interview werden insgesamt 11 Items mithilfe einer Skala von null bis zehn Punkten aus den vier folgenden Kategorien bewertet:

- Mobilität

- tägliche Aktivitäten

- Rolle in der Familie

- Selbstversorgung durch den Patienten Es können aus statistischer Sicht 100 Punkte erreicht werden. Die Formel zur Berechnung der Punktzahl des RNL-I ist sehr einfach. Die Summe des RNL-I wird durch 110 dividiert und anschließend mit 100 multipliziert.

Die Interpretation des RNL-I gestaltet sich etwas schwieriger. Einfach zu sehen ist, dass eine niedrige Punktzahl auch für eine niedrige Teilhabe und Integration steht (Tab. 9). Jedoch lässt sich nur sehr undeutlich daraus ablesen, ab welcher Punktzahl die Integration sehr gut oder deutlich schlecht ist. Hier hilft es manchmal, Vergleiche mit bereits untersuchten Krankheitsbildern anzustellen. Zum Beispiel weisen Patienten nach einem Schlaganfall eine durchschnittliche Punktzahl von 84,3 Punkten im RNL-I auf [57]. Patienten mit peripheren Lähmungen, z. B. mit auf Intensivstation erworbenem Schwächesyndrom, liegen laut den ersten vorläufigen, noch nicht veröffentlichten $\mathrm{Er}$ -

Tab. 9 Deutsche Übersetzung des RNL-I (in Anlehnung an [42]) Item \begin{tabular}{l|l|l|l|l|l|l|l|l|l|l|}
0 & 1 & 2 & 3 & 4 & 5 & 6 & 7 & 8 & 9 & 10
\end{tabular}

1. Ich bewege mich, wenn ich es für notwendig erachte, innerhalb meines Wohnviertels.

2. Ich bewege mich bei Bedarf innerhalb meiner Stadt.

3. Ich bin imstande, bei Bedarf die Stadt zu verlassen.

4. Ich bin zufrieden mit meiner Selbstversorgung wie z. B.: Anziehen, Essen, Auf-Toilette-Gehen, Waschen/ Duschen/Baden.

5. Ich verbringe die meiste Zeit des Tages bei einer Arbeit, die notwendig oder wichtig für mich ist.

6. Ich bin imstande, an Erholungsaktivitäten (Hobbys, Sport, Lesen, Computer etc.) teilzuhaben, wenn ich das möchte.

7. Ich nehme an sozialen Aktivitäten mit der Familie, Freunden und/oder Kollegen teil, wenn ich es für mich als notwendig oder wünschenswert erachte.

8. In meiner Familie nehme ich eine Rolle ein, die auf meine Bedürfnisse und auf die der Familienmitglieder abgestimmt ist.

9. Generell bin ich mit meinen persönlichen Beziehungen zufrieden.

10. Generell bin ich mit mir zufrieden, wenn ich in der Gesellschaft anderer bin.

11. Ich denke, ich kann mit Lebensereignissen/Schicksalsschlägen umgehen, wenn diese eintreten.

gebnissen einer laufenden Studie deutlich unterhalb der Punktzahl von Schlaganfallpatienten [43].

\section{Die Intrarater- und Interrater- \\ Reliabilität des RNL-I ist moderat [57]. \\ Dies kann darauf zurückzuführen sein, dass die Befragung mit dem RNL-I teilweise an den Angehörigen der Patienten und nicht an den Patienten selbst durchgeführt wurde [57]. Die Validität und die interne Konsistenz sind hingegen sehr gut [7, 25, 38]. Die Untersuchung der Reintegration mit dem RNL-I kann auch zuverlässig postalisch mittels Fragebogen durchgeführt werden [12]}

EQ-5D-3L. Ein Assessment zur Beurteilung der Lebensqualität ist der EQ-5D-3L. Dieser besteht aus den Punkten:

- Schmerzen und körperliche Beschwerden

- Angst und Niedergeschlagenheit

- Beweglichkeit und Mobilität

- alltägliche Tätigkeiten

- Selbstversorgung
Der Patient kann pro Kategorie eine von drei Antwortmöglichkeiten ankreuzen, welche fünf, zehn oder 15 Punkten entsprechen [19]. Anschließend wird der Gesundheitszustand mittels einer numerischen Skala von 0 bis 100 erfasst. Der EQ-5D-3L ist moderat valide und sehr zuverlässig [14, 20, 54].

Dieser Test kann auch mit fünf statt drei Einschätzungsmöglichkeiten durchgeführt werden. Die Langversion ist hinsichtlich der Rücklaufquote für die postalische Befragung der Kurzversion überlegen, jedoch fehlen noch Untersuchungen zu den psychometrischen Eigenschaften des Tests. Daher scheint die Kurzversion derzeitig zuverlässiger und sollte der Langversion vorgezogen werden [27]. Der Test ist lizenziert und damit bedingt gebührenpflichtig. Für den Gebrauch bedarf es einer Registrierung auf der Webseite der EuroQol-Gruppe.

Upper Extremity Motor Activity Log. Wie häufig und wie gut ein Patient z.B. nach einem Schlaganfall seine Arme im Alltag benutzt, misst der Upper Extremity Motor Activity Log (UE MAL) [5, 22, 44]. Es existieren mehrere Versionen des UE MAL, die sich 


\begin{tabular}{|l|l|}
\hline Tab. 10 Skala für Bewegungshäufigkeit \\
\hline Skala & Bewegungshäufigkeit \\
\hline 0 oder 0,5 & Der Arm wird nie benutzt. \\
\hline 1 oder 1,5 & $\begin{array}{l}\text { Der Arm wird sehr selten } \\
\text { benutzt. }\end{array}$ \\
\hline 2 oder 2,5 & $\begin{array}{l}\text { Der Arm wird manchmal } \\
\text { benutzt. }\end{array}$ \\
\hline 3 oder 3,5 & $\begin{array}{l}\text { Der Arm wird ungefähr halb so } \\
\text { häufig wie vor dem Schlagan- } \\
\text { fall benutzt. }\end{array}$ \\
\hline 4 oder 4,5 & $\begin{array}{l}\text { Der Arm wird fast so häufig } \\
\text { benutzt wie vor dem Schlag- } \\
\text { anfall. }\end{array}$ \\
\hline 5 oder 5,5 & $\begin{array}{l}\text { Der Arm wird genauso } \\
\text { häufig benutzt wie vor dem } \\
\text { Schlaganfall. }\end{array}$ \\
\hline
\end{tabular}

Tab. 11 Skala für die Bewegungsqualität

\begin{tabular}{|l|l|}
\hline Skala & Bewegungsqualität \\
\hline 0 oder 0,5 & $\begin{array}{l}\text { Der Arm kann überhaupt nicht } \\
\text { benutzt werden. }\end{array}$ \\
\hline 1 oder 1,5 & $\begin{array}{l}\text { Der Arm wird zwar bewegt, ist } \\
\text { aber kaum von Nutzen. }\end{array}$ \\
\hline oder 2,5 & $\begin{array}{l}\text { Der Arm ist von gewissem } \\
\text { Nutzen, benötigt aber Hilfe vom } \\
\text { gesunden Arm, oder er kann nur } \\
\text { sehr langsam bzw. mit Schwie- } \\
\text { rigkeiten benutzt werden. }\end{array}$ \\
\hline 3 oder 3,5 & $\begin{array}{l}\text { Der Arm wird benutzt, aber die } \\
\text { Bewegungen sind langsam oder } \\
\text { können nur mit einiger Anstren- } \\
\text { gung ausgeführt werden. }\end{array}$ \\
\hline 4 oder 4,5 & $\begin{array}{l}\text { Die Bewegungen sind fast } \\
\text { normal, aber nicht ganz so } \\
\text { schnell und genau. }\end{array}$ \\
\hline 5 oder 5,5 & $\begin{array}{l}\text { Der Arm kann so gut benutzt } \\
\text { werden wie vor dem Schlag- } \\
\text { anfall. }\end{array}$ \\
\hline
\end{tabular}

hinsichtlich der Anzahl der Fragen und demnach auch bezüglich der Durchführungsdauer unterscheiden.

Im Praxisalltag ist der UE MAL 14 mit 14 Fragen im Vergleich zum Originaltest mit 30 bis 45 einzuschätzenden Bewegungen am praktikabelsten und ist mit der guten bis sehr guten Reliabilität und Validität der Langversion vergleichbar (Tab. 13) [58]. In diesem Interview soll der Patient in 15 bis 20 min Alltagsbewegungen des Armes hinsichtlich Bewegungshäufigkeit und Bewegungsqualität mit zwei unterschiedlichen Skalen von jeweils von 0 bis 5 einschätzen (Tab. 10 u. Tab. 11). Die Anleitung des Testverfahrens gliedert sich in vier Schritte:

Tab. 12 Kodierung des Grundes für die Antwort „Nein“ in der ersten Fragerunde Kodierung für Antwort „Nein“ Punkte Häufigkeit/Qualität

Ich nutzte ausschließlich den nicht betroffenen Arm für 0 Punkte/0 Punkte diese Bewegung

Das macht immer jemand anderes für mich.

0 Punkte/0 Punkte

Ich führe diese Bewegung niemals durch. Auch wenn ich Frage streichen es könnte (z. B. Haare kämmen bei sehr kurzem Haar).

Ich habe diese Bewegung das letzte Mal vor über einer Bewertung mit Ja, Beurteilung der Woche durchgeführt. Seitdem aber nicht mehr. letzten Bewegung

Ich schreibe/esse etc. schon immer mit der nicht Frage streichen

betroffenen Hand

Tab. 13 Aufgaben- und Bewertungstabelle UE MAL 14 (nach freier deutscher Übersetzung) Aufgabe:

Schritt 1) Erklärung der Testbedingungen

Schritt 2) Ja/Nein: Haben Sie ...?

Schritt 3) Häufigkeit: Schätzen Sie mit der Häu-

figkeitsskala folgende Bewegung ein.

Wie häufig haben Sie mit dem betroffenen Arm ...?

Schritt 4) Qualität: Schätzen Sie mit der

Qualitätsskala folgende Bewegung ein.

Wie gut haben Sie mit dem betroffenen Arm ...?

1.... ein Buch gehalten?

2. ... ein Handtuch benutzt?

3.... ein Glas angehoben?

4. ... die Zähne geputzt?

5. ... sich rasiert/geschminkt?

6. ... die Tür mit dem Schlüssel geöffnet?

7. ... geschrieben/getippt?

8. ... generell selbstständig bewegt?

9. ... den Arm durch ein Kleidungsstück geführt?

10. ... etwas getragen?

11. ... Besteck gehalten?

12. ... Haare gekämmt?

13. ... eine Tasse am Henkel angehoben?

14. ... Kleidung zugeknöpft?

- Schritt 1: Die Skalen und der Test müssen dem Patienten vor der Untersuchung ausführlich erklärt werden.

- Schritt 2: Der Patient soll bestimmte Fragen mit „Ja“ oder „Nein“ beantworten, die die Nutzung des Armes im Alltag widerspiegeln (Tab. 13). Wichtig ist laut Taub, dass es sich um tatsächliche Armbewegungen und nicht um die mögliche Nutzung des Armes im Alltag handelt. Beantwortet der Patient eine Frage des Tests mit „Nein“, so muss mit einem Kodex abgelesen werden, ob die Frage für die Skalenbewertung berücksichtigt und mit 0 Punkten bewertet oder aus dem Interview gestrichen wird (Tab. 12).
- Schritt 3: Der Patient soll nun mit der Skala für Bewegungshäufigkeit einschätzen, wie oft er seinen Arm bei den genannten Aufgaben, die zuvor mit Ja beantwortet wurden, benutzt. Anschließend beantwortet der Patient die Fragen nach der bestmöglichen Ausführung der Bewegung. Dafür wird die Skala für Bewegungsqualität verwendet. Der Patient hat die Möglichkeit, zwischen zwei Punktwerten, mit einem Unterschied von jeweils 0,5 Punkten, pro Beschreibung die Bewegung zu beurteilen. Die Fragestellung sollte nach Taub und Kollegen hinsichtlich des Zeitpunkts der Testung variieren. So sollte zum ersten Messzeitpunkt vor einer Therapie die Formulierung „in der letzten 
Woche“ genutzt werden, z. B.: „Haben Sie in der letzten Woche mit dem betroffenen Arm ein Buch gehalten?“ Bei der ReUntersuchung nach dem Training ist die Formulierung „seit der letzten Befragung mit diesem Test“ zu nutzen, z.B.: „Wie gut haben Sie, seit der letzten Befragung mit diesem Test, ein Buch gehalten?"

- Schritt 4: Bei jeder Antwort zu Schritt 3 soll der Untersucher den gewählten Punktwert durch die Beschreibung noch einmal wiederholen, um dem Patienten die Antwort zu spiegeln. Sollte sich der Patient darauf für eine andere Antwort entscheiden, wird der vorherige Punktwert ignoriert. Außerdem kann sich der Tester die Bewegungen vom Patienten demonstrieren lassen und zusammen mit dem Patienten einen Punktwert ermitteln, wenn dieser es wünscht. Ist der Patient auch bei Diskrepanzen von seiner eigenen Bewertung überzeugt, gilt diese als Maßstab. Weiterhin empfehlen Taub und Kollegen bei längeren Zeitabständen, z. B. bei einer Nachuntersuchung nach sechs Monaten, ein Video (im Original UE MAL How Well Rating Scale Video) mit typischen Armbewegungen zu zeigen, um die Patienten auf die Bewertung der Bewegungsqualität zu sensibilisieren.

Die Summe des Tests ergibt sich aus der addierten Gesamtpunktzahl dividiert durch die Anzahl der Fragen. Je höher die Punktzahl, desto häufiger wird der Arm mit entsprechender Bewegungsqualität eingesetzt.

\section{Fazit}

Alle aufgeführten Assessments weisen deutliche Stärken, aber auch Schwächen auf. Es liegt am Therapeuten, den passenden Test für die richtigen Umstände zu finden. Aus eigener Erfahrung können folgende fünf Fragestellungen bei der Wahl des richtigen Tests helfen:

- Welches Assessment testet vollständig die Symptomatik, die ich behandeln oder evaluieren möchte?

- Kann der Test nach wiederholter Untersuchung Veränderungen objektiv sichtbar machen?

- Ist der Test vollständig vom Patienten durchführbar?

- Habe ich die Mittel, Zeit, Raum und genaues Wissen, um den Test anzuwenden?

- Könnte ein anderer Therapeut die Messung definitiv mit dem gleichen Ergebnis ohne bedeutende Abweichungen ausführen?

\section{Autoren

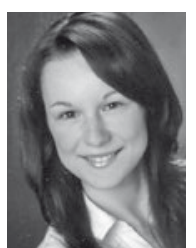 \\ Simone Thomas ist Physiothera- peutin mit Bachelor und Master of Science. Seit 2012 unterstützt sie das Wissenschaftliche Institut der Klinik Bavaria Kreischa als wissenschaftliche Mitarbeiterin. \\ Simone Thomas}

Wissenschaftliche Mitarbeiterin

Private Europäische Medizinische Akademie der Klinik Bavaria in Kreischa $\mathrm{GmbH}$

An der Wolfsschlucht 1-2

01731 Kreischa

E-Mail: simone.thomas@klinik-bavaria.de

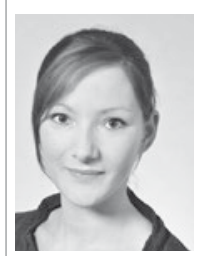

Bettina Scheffler ist als Physiotherapeutin in der Klinik Bavaria in Kreischa, Bereich Neurologie, tätig. Sie ist Absolventin des Bachelorstudiengangs Physiotherapie an der SRH Hochschule für Gesundheit, Gera, und studiert derzeit im

Masterstudiengang Neurorehabilitation. Bettina Scheffler Kantstraße 3 01187 Dresden

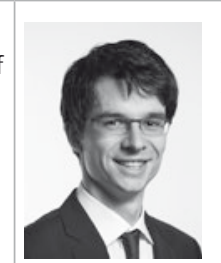

Bernhard Elsner ist Physiotherapeut und Professor für Therapiewissenschaften an der SRH Fachhochschule für Gesundheit in Gera. Er ist Studiengangleiter der Studiengänge Physiotherapie und Ergotherapie.

Prof. Dr. Bernhard Elsner, MPH

Professor für Therapiewissenschaften

SRH Hochschule für Gesundheit Gera $\mathrm{gGmbH}$

Neue Str. 28-30

07548 Gera

Website: www.srh-gesundheitshochschule.de E-Mail: bernhard.elsner@srh-gesundheitshochschule.de

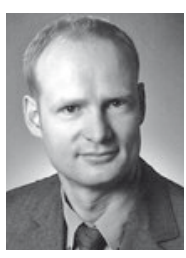

Jan Mehrholz ist Physiotherapeut und Professor für Therapiewissenschaften. Zudem leitet er das Wissenschaftliche Institut der Klinik Bavaria in Kreischa und ist als Privatdozent für Gesundheitswissenschaften/Public Health an der Medizinischen Fakultät der TU Dresden tätig.

Prof. Dr. rer. medic. habil. Jan Mehrholz Leiter Wissenschaftliches Institut Private Europäische Medizinische Akademie der Klinik Bavaria in Kreischa GmbH An der Wolfsschlucht 1-2 01731 Kreischa
Die komplette Literaturliste finden Sie unter www.thieme-connect.de/ejournals

Bibliografie

DOI 10.1055/s-0042-105775

neuroreha 2016; 8: 76-85

(c) Georg Thieme Verlag KG

Stuttgart · New York · ISSN 1611-649 